\title{
Flow Properties Inferred from Generalized Maxwell Models
}

\author{
Siegfried Hess ${ }^{\mathrm{a}}$, Bastian Arlt ${ }^{\mathrm{a}}$, Sebastian Heidenreich $^{\mathrm{a}}$, Patrick Ilg ${ }^{\mathrm{a}, \mathrm{b}}$, Chris Goddard ${ }^{\mathrm{c}}$, \\ and Ortwin Hess ${ }^{\mathrm{c}}$ \\ ${ }^{a}$ Institut für Theoretische Physik, Technische Universität Berlin, EW 7-1, Hardenbergstraße 36, \\ D-10623 Berlin, Germany \\ b Institut für Polymere, ETH Zürich, HCI H 541, Wolfgang Pauli Str. 10, CH-8093 Zürich, \\ Switzerland \\ c Advanced Technology Institute, School of Electronics and Physical Sciences, University of Surrey, \\ Guildford, GU2 7XH, UK
}

Reprint requests to S. H.; E-mail: S.Hess@ physik.tu-berlin.de

Z. Naturforsch. 64a, 81 - 95 (2009); received September 2, 2008

\begin{abstract}
The generalized Maxwell model is formulated as a nonlinear relaxation equation for the symmetric traceless stress tensor. The relaxation term of the equation involves the derivative of a potential function with respect to the stress tensor. Two special cases for this potential referred to as "isotropic" and "anisotropic" are considered. In the first case, the potential solely depends on the second scalar invariant, viz. the norm of the tensor. In the second case, also a dependence on the third scalar invariant, essentially the determinant, is taken into account in analogy to the Landau-de Gennes potential of nematic liquid crystals. Rheological consequences of the model are presented for a plane Couette flow with an imposed shear rate. The non-Newtonian viscosity and the normal stress differences are analyzed for stationary solutions. The dependence on the model parameters is discussed in detail. In particular, the occurrence of a shear-thickening behaviour is studied. The possibility to describe substances with yield stress and the existence of non-stationary, stick-slip-like solutions are pointed out. The extension of the model to magneto-rheological fluids is indicated.
\end{abstract}

Key words: Generalized Maxwell Model; Non-Newtonian Viscosity; Normal Stress Differences; Shear-Thinning and Shear-Thickening; Stick-Slip Behaviour; Magneto-Rheological Behaviour.

\section{Introduction}

The generalized Maxwell model is a nonlinear extension of the standard corotational or codeformational Maxwell model equation governing the symmetric traceless (deviatoric) friction stress tensor. The relaxation term involves the derivative of a potential function with respect to the stress tensor. In general, this potential depends on the second- and third-order invariants $I_{2}$ and $I_{3}$ of the stress tensor which are essentially the square of the magnitude (norm) and the determinant of the tensor, respectively. In the ordinary Maxwell model, the potential is linear in $I_{2}$ and the Maxwell model equation is linear in the stress tensor. The nonlinear generalization was invented in order to treat not only shear-thinning but also shear-thickening behaviour [1], i. e. the increase of the viscosity with increasing shear rate as it occurs in dense colloidal dispersions [2-4] and in some polymeric fluids [5], as well as in surfactant solutions $[6,7]$. The model can also be applied to systems with yield stress. For certain values of the relevant parameters periodic stickslip-like solutions are found and even a rather complex chaotic rheological behaviour occurs for a simple flow geometry [8]. In the applications of the nonlinear Maxwell model analyzed so far, the potential function was chosen in analogy to the Landau-de Gennes potential used in a dynamic theory for the flow alignment in the isotropic and nematic phases of liquid crystals [919]. The Landau-de Gennes expression contains terms linear and quadratic in $I_{2}$ and a term linear in $I_{3}$. Here we study the consequences of an alternative potential function involving terms of first, second and third order in $I_{2}$ but no terms depending on $I_{3}$. This type of potential is referred to as "isotropic" since it solely depends on the magnitude of the deviatoric stress tensor. Yet again, a rather complex rheological behaviour is found which includes shear-thickening as well as the occurrence of a yield stress and a non-stationary response to an imposed stationary shear rate for certain 
ranges of the relevant model parameters. A comparison with results based on the previous "anisotropic" potential function is appropriate. The qualitatively different types of the rheological behaviour are correlated with the relevant model parameters.

The thermodynamic modeling of equilibrium properties and non-equilibrium phenomena in complex fluids and solids requires the treatment of internal variables and their coupling with the standard variables of thermo-hydrodynamics. In addition to general principles [20] the tensorial character of the internal variables has to be taken into account for specific applications, e. g., a vectorial variable is needed for dielectric relaxation [21] and the treatment of polarizable media [22]. In this article, second-rank tensorial variables are considered. Inspired by the equations governing the alignment tensor of nematic liquid crystals, the focus is on the extra stress tensor which obeys a nonlinear Maxwell model equation. It provides a generic model for the treatment of rheological properties. A generalization where the coupling between the tensorial variable and the electric polarization vector has been taken into consideration has been presented recently [23]. Here modifications needed to describe magneto-rheological fluids are discussed briefly.

This article proceeds as follows. Firstly, the equation for the nonlinear Maxwell model is stated. The relaxation equation for the symmetric traceless stress tensor contains the derivative of a potential function with respect to this tensor. The general form of the potential is discussed and the two special cases referred to as "isotropic" and "anisotropic" potentials are presented. For convenience, scaled variables are introduced. Then, in the following analysis, a plane Couette flow is considered, with a given shear rate. Basis tensors are used and the nonlinear tensorial relaxation equation is rewritten in terms of five coupled equations for the five components of the stress tensor. One of these components is the shear stress which serves to define the non-Newtonian viscosity, two other ones are associated with the first and second normal stress differences and hence with the viscometric functions. The remaining two components break the plane Couette symmetry, and they relax to zero in many cases, but not always. The main part of this article is devoted to properties of stationary solutions, in particular to their dependence on the model parameters. Implicit analytical solutions are given for the non-Newtonian viscosity and the normal stress differences. A "shear-thickening phase diagram", in the parameter space, is inferred from the dependence of the viscosity on the shear rate. Similarly, the dependence of the first and second viscometric functions on the model parameters is discussed. A comparison with experimental data is made for one particular shear-thickening fluid. After some remarks on solutions of the model equations which describe substances with yield stress, it is pointed out that also solutions with non-stationary shear stress are possible even when the imposed shear rate is stationary. These correspond to stick-slip-like motions. Conditions for their occurrence are given. Two examples for a non-stationary response are shown. Finally, it is indicated how the theory can be extended to magnetorheological fluids.

\section{The Model Equations}

\subsection{Basics}

The symmetric traceless (deviatoric) part ' $\overline{\boldsymbol{\sigma}}$ ' of the stress tensor $\boldsymbol{\sigma}$ is decomposed into a contribution associated with the "internal structure", for which the nonlinear Maxwell model equations are formulated, and a contribution linked with a "second Newtonian" viscosity $\eta_{\infty}$ reached at high shear rates:

$$
\overline{\boldsymbol{\sigma}}^{\prime}=\sqrt{2} G \boldsymbol{\pi}+2 \eta_{\infty} \Gamma .
$$

Here $\boldsymbol{\Gamma}={ }^{\prime} \overline{\nabla v}$ ' is the deformation rate tensor, viz. the symmmetric traceless part of the velocity gradient tensor. The quantity $G$ is a reference value for a shear modulus such that $\boldsymbol{\pi}$ is a dimensionless "friction stress tensor". An obvious choice for $G$ (which is not needed now) is either the high-frequency shear modulus or the reference pressure $p_{\text {ref }}=n k_{\mathrm{B}} T$, where $n$ and $T$ are the number density and the temperature. The factor $\sqrt{2}$ has been inserted for convenience, the factor 2 in (1) is conventional. The symbol ' $\cdots$ ' indicates the symmetric traceless part of a tensor, e. g. in Cartesian tensor notation, ${ }^{\prime \sigma_{\mu v}}=\frac{1}{2}\left(\sigma_{\mu v}+\sigma_{v \mu}\right)-\frac{1}{3} \sigma_{\lambda \lambda} \delta_{\mu v}$, where $\delta_{\mu v}$ is the unit tensor.

When $\boldsymbol{\pi}$ obeys the Maxwell model equation, the constitutive relation (1) is referred to as Jeffrey model. In the following a generalization of the Maxwell model is used. When the tensor $\boldsymbol{\pi}$ is interpreted as the second moment of the velocity distribution function of a gas, an equation of this type can be and has been derived from the nonlinear Boltzmann equation [24, 25]. For a simple liquid, the dominant contribution to the friction stress is the virial tensor for which a generalized 
Maxwell equation can be derived from a kinetic equation of Kirkwood-Smoluchowski type for the pair correlation function [26]. For polymeric fluids, $\boldsymbol{\pi}$ can be linked with the symmetric traceless part of the secondrank conformation tensor [16,27].

It should be stressed that the present approach is not dealing with the liquid crystal problem but with a case where the stress tensor is the sum of two contributions each of which obeys its own constitutive equation. Even for a simple fluid, it is well known from microscopic theory, that the pressure tensor or stress tensor is the sum of kinetic and potential (configurational) contributions whose dynamics can be derived from kinetic equations for the velocity distribution function and the pair correlation function, respectively. In both cases, equations similar to (2) can and have been derived. We assume that equations of similar kind can be used for complex fluids where a simple microscopic theory is not available. The ansatz (1) differs from that one used in liquid crystal theory where the second-rank alignment tensor (also referred to as order parameter tensor, Q-tensor, S-tensor) is treated as an additional macroscopic variable which is coupled with the stress tensor. Derivations of the equations relevant for liquid crystals and applications were given in [9-15,28-31].

\subsection{Relaxation Equation for the Stress Tensor}

The friction stress tensor is assumed to obey the generalized Maxwell equation $[1,8]$

$$
\frac { \partial \boldsymbol { \pi } } { \partial t } - 2 \longdiv { \omega \times \boldsymbol { \pi } } - 2 \kappa \widehat { \boldsymbol { \Gamma } \cdot \boldsymbol { \pi } } + \tau _ { 0 } ^ { - 1 } \boldsymbol { \Phi } ( \boldsymbol { \pi } ) = \sqrt { 2 } \boldsymbol { \Gamma } .
$$

Here $\tau_{0}$ is a relaxation time coefficient. The tensor $\boldsymbol{\Phi}=\partial \Phi / \partial \boldsymbol{\pi}$ is the derivative of a "potential" function $\Phi$ with respect to the deviatoric stress tensor $\pi$. The scalar function $\Phi=\Phi\left(I_{2}, I_{3}\right)$ depends on the second and third scalar invariants, written in component notation, $I_{2}=\pi_{\mu v} \pi_{\mu v}, I_{3}=\sqrt{6} \pi_{\mu v} \pi_{v \lambda} \pi_{\lambda \mu}=$ $3 \sqrt{6} \operatorname{det}(\pi)$. The second term gives the coupling between the vorticity $\omega$ and the tensor $\boldsymbol{\pi}$. The term in (2) involving the cross-product $x$ of the vorticity with the stress tensor reads, in cartesian tensor notation, $2(\omega \times \pi)_{\mu v}=\varepsilon_{\mu \lambda \rho} \omega_{\lambda} \pi_{\rho v}+\varepsilon_{v \lambda \rho} \omega_{\lambda} \pi_{\rho \mu}$.

The "linear" Maxwell model pertains to the simplest choice for the potential function $\Phi$, viz. $\Phi=$ $\frac{1}{2} A I_{2}$, with a dimensionless coefficient $A>0$. This implies $\boldsymbol{\Phi}=A \boldsymbol{\pi}$. For $\boldsymbol{\kappa}=0$ the time change in (2) is governed by the corotational time derivative. Thus the relaxation equation (2) reduces to the Jaumann-Maxwell model with the Maxwell relaxation time $\tau=\tau_{0} A^{-1}$. The Newtonian viscosity, attained for small shear rates is $\eta=\eta_{\text {New }}:=G \tau+\eta_{\infty}=G \tau_{0} A^{-1}+\eta_{\infty}$.

For $\kappa=1$ or $\kappa=-1$ the time change corrersponds to that of a codeformational time derivative. In analogy to the corresponding equation for the alignment tensor of nematics [10], $\kappa$ is regarded as an additional model parameter. For $\kappa \neq 0, \pm 1$ and a linear relaxation term, (2) reduces to the Johnson-Segalman model [32-34].

A corresponding term appears in the equation for the potential (configurational) part of the stress tensor derived from a kinetic equation for the pair correlation function of a simple fluid [35]. For a special case of a fluid composed of particles interacting with a power law binary potential proportional to $r^{v}$ ( $r$ is the distance between two particles), $\kappa=3 / 7+(2 / 3) v$. Thus one has e. $g . \kappa=1$ and $\kappa=-3$ for a harmonic oscillator $(v=2)$ and for "soft spheres" $(v=-12)$. Notice that the magnitude of $\kappa$ may be larger than 1 .

In the absence of flow the stationary solution of (2) is given by $\boldsymbol{\Phi}=0$. In a fluid state one has $\boldsymbol{\pi}=0$ and this is a stable solution corresponding to the absolute minimum of the potential function $\Phi$. The quantity $\Phi$ is a nonlinear function of the stress and the equation $\boldsymbol{\Phi}=0$ might also have solutions with $\boldsymbol{\pi}$ different from zero for certain ranges of the model parameters. If such a solution is (locally) stable the system possesses a yield stress.

\subsection{Potential Function}

When terms up to sixth order in the stress tensor are taken into consideration the ansatz for the potential function is

$$
\Phi=\frac{1}{2} A I_{2}-\frac{1}{3} B I_{3}+\frac{1}{4} C I_{2}^{2}+\frac{1}{5} D I_{2} I_{3}+\frac{1}{6} E I_{2}^{3}+\frac{1}{6} F I_{3}^{2},
$$

where the dimensionless coefficients $A, B, C, D, E, F$ are model parameters. Notice that the derivatives of $I_{2}$ and $I_{3}$ with respect to $\pi_{\mu v}$ are, in component notation, $2 \pi_{\mu \nu}$ and $3 \sqrt{6}{ }^{\prime} \pi_{\mu \lambda} \pi_{\lambda v}$, respectively.

A potential function which imposes a limiting magnitude of the stress tensor has been stated in [8]. Consequences of a similar expression for the potential governing the alignment tensor of nematic liquid crystals has been studied in [28]. Compared with the simpler choice (3), quantitative but practically no qualitative differences are found. This point is not considered here. 
In analogy to the equations governing the alignment tensor of nematic liquid crystals, the simple ansatz with terms up to fourth order in the stress tensor was made previously $[1,8]$. This means that $D=E=F=0$ and $C>0$. Then one has

$$
\boldsymbol{\Phi}=A \boldsymbol{\pi}-\sqrt{6} B^{\prime} \overline{\boldsymbol{\pi} \cdot \boldsymbol{\pi}}{ }^{\prime}+C \boldsymbol{\pi}(\boldsymbol{\pi}: \boldsymbol{\pi}) .
$$

The model introduced by Giesekus [36] for the study of polymeric fluids corresponds to $B \neq 0, C=D=$ $E=F=0$. For a discussion of related models see [16], p. 173.

Here the consequences of the alternative choice $B=$ $D=F=0$ with $C<0$ and $E>0$ are analyzed. In this case

$$
\boldsymbol{\Phi}=\boldsymbol{\pi}\left(A+C(\boldsymbol{\pi}: \boldsymbol{\pi})+E(\boldsymbol{\pi}: \boldsymbol{\pi})^{2}\right)
$$

holds true instead of (4). Where appropriate, the results based on the isotropic potential are compared with those which follow from the previously used anisotropic potential.

For the special case where the stress tensor is uniaxial the ansatz $\boldsymbol{\pi}=\sqrt{\frac{3}{2}} \pi_{0}{ }^{\prime \mathbf{n n}}$ can be made. Here $\mathbf{n}$ is a unit vector parallel to the symmetry axis. In this case $\boldsymbol{\Phi}=0$ as given by (4) corresponds to $\pi_{0}(A-$ $\left.B \pi_{0}+C \pi_{0}^{2}\right)=0$. Solutions of this equation are $\pi_{0}=0$, corresponding to a fluid state, and $\pi_{0}=B /(2 C) \pm$ $\sqrt{B^{2} /\left(4 C^{2}\right)-A / C}$, if $A \leq B^{2} /(4 C)$. For the special case of a planar biaxial stress tensor, viz. for $\boldsymbol{\pi}=$ $\frac{1}{2} \sqrt{2} \pi_{1}\left(\mathbf{e}^{x} \mathbf{e}^{x}-\mathbf{e}^{y} \mathbf{e}^{y}\right)$, with orthogonal unit vectors $\mathbf{e}^{x}$, $\mathbf{e}^{y}, \boldsymbol{\Phi}=0$ as given by (4) implies $\pi_{1}\left(A+C \pi_{1}^{2}\right)=0$. Here solutions are $\pi_{1}=0$, corresponding to a fluid state, and $\pi_{1}= \pm \sqrt{-A / C}$, if $A<0$. The cases $\pi_{0} \neq 0$ or $\pi_{1} \neq 0$ in the absence of a flow correspond to a (metastable) solid state with a yield stress. The potential function is anisotropic in the space spanned by the five components of the tensor. This is different for the case considered next.

The solution corresponding to $\boldsymbol{\Phi}=0$ for (5) applies for all components of the stress tensor, irrespective whether it has uniaxial or biaxial symmetry. This is due to the fact that the underlying "isotropic" potential function does not depend on the invariant $I_{3}$. Here, one has $\pi=0$ or $\pi^{2}=-C /(2 A) \pm \sqrt{C^{2} /\left(4 A^{2}\right)-E / A}$, if $A \neq 0, C^{2}>4 E A$, and $\pi^{2}=(\boldsymbol{\pi}: \boldsymbol{\pi})>0$.

In the Landau theory for (equilibrium) phase transitions the assumption is made that $A$ depends on the temperature $T$ or on the density $\rho$ according to $A=$ $A_{0}\left(1-T_{0} / T\right)$ or $A=A_{0}\left(1-\rho / \rho_{0}\right)$ with characteristic temperature $T_{0}$ or density $\rho_{0}$. Here such a specific dependence of $A$ is not needed, but it is presupposed that $A$ decreases with decreasing temperature and increasing density.

\subsection{Scaled Variables}

Scaled variables are introduced in order to characterize the importance of the terms nonlinear in the stress by a single model parameter. In previous studies based on (4) (cf. [1,8]) the procedure used for the isotropic-nematic phase transition in liquid crystals $[14,28]$ was followed. This means that the components of $\boldsymbol{\pi}$ are expressed in units of $\pi_{c}=\frac{2}{3} \frac{B}{C}, \boldsymbol{\pi}=$ $\pi_{c} \pi^{*}$. With $A=A^{*} A_{c}, A_{c}:=2 B^{2}(9 C)^{-1}, \tau_{c}=\tau_{0} A_{c}^{-1}=$ $\tau_{0}\left(\frac{1}{2} C \pi_{C}^{2}\right)^{-1}, C>0$, and $t=\tau_{c} t^{*}, \Gamma_{\mu \nu}^{*}=\tau_{c} \Gamma_{\mu \nu}$, the model coefficients $B$ and $C$ no longer appear explicitely in the relaxation equations. The scaled variables are denoted by the same symbol as the original variables when no danger of confusion is expected, e. g. $\pi_{\mu \nu}^{*} \rightarrow$ $\pi_{\mu \nu}, t^{*} \rightarrow t$. Then we have, instead of (4),

$$
\boldsymbol{\Phi} \rightarrow A^{*} \boldsymbol{\pi}-3 \sqrt{6}^{\prime} \overline{\boldsymbol{\pi} \cdot \boldsymbol{\pi}}+2 \boldsymbol{\pi}(\boldsymbol{\pi}: \boldsymbol{\pi}) .
$$

For the special case of a uniaxial stress the scaled potential function is $\Phi(\pi)=\frac{1}{2} A^{*} \pi_{0}^{2}-\pi_{0}^{3}+\frac{1}{2} \pi_{0}^{4}$, and $\boldsymbol{\Phi}=$ 0 corresponds to $\pi_{0}\left(A^{*}-3 \pi_{0}+2 \pi_{0}^{2}\right)=0$. For $A^{*}=1$ one has "phase coexistence", i. e. $\Phi(0)=\Phi(1)$.

For the isotropic potential to be studied here a different scaling is introduced. Again, one writes $\boldsymbol{\pi}=\pi_{c} \boldsymbol{\pi}^{*}$. But now, subject to $C<0, \pi_{c}$ is defined by

$$
\pi_{c}=\frac{1}{2} \sqrt{\frac{-3 C}{E}} .
$$

With

$$
\begin{aligned}
& A=A^{*} A_{c}, \quad A_{c}:=3 C^{2}(16 E)^{-1}, \quad \tau_{c}=\tau_{0} A_{c}^{-1}, \\
& E>0
\end{aligned}
$$

times and shear rates expressed in units of $\tau_{c}$ and $\tau_{c}^{-1}$, respectively, the model coefficients $C$ and $E$ no longer appear explicitely in the relaxation equations. The scaled variables are denoted by the same symbol as the original variables when no danger of confusion is expected, e. g. $\pi_{\mu \nu}^{*} \rightarrow \pi_{\mu \nu}, t^{*} \rightarrow t$. Then we have, instead of (5),

$$
\boldsymbol{\Phi} \rightarrow \boldsymbol{\pi}\left(A^{*}-4(\boldsymbol{\pi}: \boldsymbol{\pi})+3(\boldsymbol{\pi}: \boldsymbol{\pi})^{2}\right) .
$$


The coefficient $A^{*}$ determines whether terms of higher order in the components of the pressure tensor are of relevance $\left(A^{*} \approx 1\right)$ or not $\left(A^{*} \gg 1\right)$. It is assumed that $A^{*}$ depends strongly on the temperature $T$ or on the number density $\rho$ of a dispersion in a way typical for a Landau-type theory of a phase transition. More specifically, it is expected that $A^{*}$ decreases with decreasing $T$ or with increasing $\rho$. The Newtonian viscosity increases with decreasing $T$ or increasing $\rho$ due to the increase of the relaxation time $\tau$. The fluid state "coexists" with a state possessing a yield stress at $A^{*}=1$. For the dynamics based on (6) or (9), no stationary solutions with non-zero values of the tensor $\pi$ exist in equilibrium, if $A^{*}>A_{\mathrm{s}}$, with $A_{\mathrm{s}}=9 / 8=1.125$ or $A_{\mathrm{S}}=4 / 3 \approx 1.333$, respectively.

\section{Plane Couette Geometry}

\subsection{Basis Tensors}

In general, the symmetric traceless shear stress tensor has five independent components. For a plane Couette flow with the velocity in $x$-direction, its gradient in $y$-direction, and the vorticity in $z$-direction, it is convenient to decompose the stress tensor with respect to the orthonormalized tensors $\boldsymbol{T}^{k}, k=0, \ldots, 4$ (cf. [37]) according to $\boldsymbol{\pi}=\sum_{k=0}^{4} \pi_{k} \boldsymbol{T}^{k}$, with $\boldsymbol{T}^{0} \equiv \sqrt{3 / 2} \mathbf{e}^{z} \mathbf{e}^{z}$, $\boldsymbol{T}^{1} \equiv \sqrt{1 / 2}\left(\mathbf{e}^{x} \mathbf{e}^{x}-\mathbf{e}^{y} \mathbf{e}^{y}\right), \boldsymbol{T}^{2} \equiv \sqrt{2} \overline{\mathbf{e}^{x} \mathbf{e}^{y}}, \boldsymbol{T}^{3} \equiv \sqrt{2} \mathbf{e}^{x} \mathbf{e}^{z}$, $\boldsymbol{T}^{4} \equiv \sqrt{2} \overline{\mathbf{e}^{y} \mathbf{e}^{z}}$, where $\mathbf{e}^{x \cdot y \cdot z}$ are unit vetors parallel to the coordinate axes. The orthogonality relation and the expression for the coefficients $\pi_{k}$ are given by $\boldsymbol{T}^{i}: \boldsymbol{T}^{k}=$ $\delta_{i k}$ and $\pi_{k}=\boldsymbol{\pi}: \boldsymbol{T}^{k}$. For a plane Couette geometry it frequently suffices to consider the three components $\pi_{2}$, $\pi_{1}, \pi_{0}$. For the plane Couette geometry, the shear stress is essentially $\pi_{2}$ whereas the coefficients $\pi_{1}$ and $\pi_{0}$ are associated with normal stress differences. The components $\pi_{3}$ and $\pi_{4}$ are referred to as symmetry-breaking components.

\subsection{Viscosity and Stress Components}

The non-Newtonian viscosity $\eta$ is the ratio of the shear stress and the applied shear rate, thus

$$
\sigma_{x y}=\eta \dot{\gamma}=G H \Gamma
$$

Here $\Gamma$ is the shear rate $\dot{\gamma}=\partial v_{x} / \partial y$ multiplied by the reference relaxation time $\tau_{c}$ and $v_{x}$ is the $x$-component of the flow velocity. In the following, results for the viscosity are presented in units of the reference viscosity $\eta_{\text {ref }}=G \tau_{c}$. The dimensionless viscosity coefficient $H$ is determined by

$$
H=\pi_{c} \pi_{2} / \Gamma+H_{\infty}, \quad H_{\infty}=\eta_{\infty} / \eta_{\text {ref }} .
$$

Similarly, the scaled (dimensionless) shear stress

$$
\sigma^{*}=H \Gamma=\pi_{c} \pi_{2}+H_{\infty} \Gamma
$$

and the scaled (dimensionless) first and second normal stress differences

$$
\begin{aligned}
& N_{1}^{*}=\left(\sigma_{x x}-\sigma_{y y}\right) / G=2 \pi_{c} \pi_{1}, \\
& N_{2}^{*}=\left(\sigma_{y y}-\sigma_{z z}\right) / G=-\pi_{c}\left(\pi_{1}+\sqrt{3} \pi_{0}\right)
\end{aligned}
$$

are used. In graphs $\sigma^{*}$ and $N_{1}^{*}$ are also denoted by $\sigma$ and $v$. The viscometric functions $\Psi_{1}, \Psi_{2}$ are defined as the ratio of the normal stress differences $N_{1}, N_{2}$ and the shear rate squared. For the corresponding scaled quantities one has

$$
\Psi_{i}^{*}=N_{i}^{*} / \Gamma^{2}, \quad i=1,2 .
$$

No stationary solution with finite stress can exist in the absence of a flow for $A^{*}>A_{\mathrm{s}}$. For very small shear rates, the stationary value of the dimensionless viscosity approaches its Newtonian limit

$$
H=H_{\mathrm{New}}:=1 / A^{*}+H_{\infty},
$$

provided that $A^{*}>A_{\mathrm{S}}$.

\subsection{Relaxation Equations for the Components}

Using these basis tensors, we obtain a system of five ordinary differential equations from (2), viz.

$$
\begin{aligned}
& \dot{\pi}_{2}-\Omega \pi_{1}+\phi_{2}=\pi_{c}^{-1} \Gamma-\tilde{\kappa} \Gamma \pi_{0}, \\
& \dot{\pi}_{1}+\Omega \pi_{2}+\phi_{1}=0, \\
& \dot{\pi_{0}}+\phi_{0}=-\tilde{\kappa} \Gamma \pi_{2}, \\
& \dot{\pi_{3}}+\frac{1}{2} \Omega \pi_{4}+\phi_{3}=\frac{1}{2} \kappa \Gamma \pi_{4}, \\
& \dot{\pi_{4}}-\frac{1}{2} \Omega \pi_{3}+\phi_{4}=\frac{1}{2} \kappa \Gamma \pi_{3} .
\end{aligned}
$$

Here the dot denotes the differentiation with respect to $t^{*}$, and $\Omega$ is (twice) the dimensionless vorticity. For the plane Couette flow one has $\Omega=-\Gamma$. The abbreviation $\tilde{\kappa}=\kappa / \sqrt{3}$ is used. 
The relaxation term based on (6) leads to

$$
\begin{aligned}
\phi_{2} & =\psi_{1} \pi_{2}-3 \sqrt{3} \pi_{3} \pi_{4}, \\
\phi_{1} & =\psi_{1} \pi_{1}-\frac{3}{2} \sqrt{3}\left(\pi_{3}^{2}-\pi_{4}^{2}\right), \\
\phi_{0} & =\psi_{0} \pi_{0}+3\left(\pi_{1}^{2}+\pi_{2}^{2}\right)-\frac{3}{2}\left(\pi_{3}^{2}+\pi_{4}^{2}\right), \\
\phi_{3} & =\psi_{0} \pi_{3}-3 \sqrt{3}\left(\pi_{1} \pi_{3}+\pi_{2} \pi_{4}\right), \\
\phi_{4} & =\psi_{0} \pi_{4}-3 \sqrt{3}\left(\pi_{2} \pi_{3}-\pi_{1} \pi_{4}\right),
\end{aligned}
$$

with

$$
\begin{aligned}
& \psi_{1}=A^{*}+6 \pi_{0}+2 \pi^{2}, \\
& \psi_{0}=A^{*}-3 \pi_{0}+2 \pi^{2},
\end{aligned}
$$

where $\pi^{2}=\pi_{0}^{2}+\pi_{1}^{2}+\pi_{2}^{2}+\pi_{3}^{2}+\pi_{4}^{2}$.

The relaxation term for the isotropic potential function, i. e. that one based on (9), is considerably simpler, viz.

$$
\phi_{i}=\psi \pi_{i}, \quad i=0, \ldots, 4,
$$

with

$$
\psi=A^{*}-4 \pi^{2}+3 \pi^{4}
$$

Solutions which do not break the symmetry of the plane Couette geometry are characterized by the three components of the stress tensor occurring in (11) and (13), and one has $\pi_{3}=\pi_{4}=0$. The in-plane components can be written as

$$
\pi_{1}=p \cos (2 \chi), \quad \pi_{2}=p \sin (2 \chi),
$$

with $p^{2}=\pi_{1}^{2}+\pi_{2}^{2}$. The angle $\chi$ determines the direction of the principal axes within the $x y$-plane. In this case one infers from the first and second equations of (16):

$$
\begin{array}{r}
p(2 \dot{\chi}-\Omega)=\pi_{c}^{-1} \Gamma \cos (2 \chi)\left(1-\tilde{\kappa} \pi_{c} \pi_{0}\right), \\
\frac{1}{2}\left(p^{2}\right)+\phi_{1} \pi_{1}+\phi_{2} \pi_{2}=\pi_{c}^{-1} \Gamma p \sin (2 \chi)\left(1-\tilde{\kappa} \pi_{c} \pi_{0}\right) .
\end{array}
$$

It is recalled that $\Omega=-\Gamma$ for the plane Couette flow. The case $\Omega=0$ corresponds to a vorticity free planar biaxial flow as realized in the four-roller geometry. For a stationary Couette flow (22) leads to

$$
\cos (2 \chi)=\pi_{c} p\left(1-\tilde{\kappa} \pi_{c} \pi_{0}\right)^{-1} .
$$

Of course, the condition $\pi_{c} p<1-\tilde{\kappa} \pi_{c} \pi_{0}$ has to be fulfilled for the stationary solution to exist. Consequences of (24) and of the stationary version of (23) for the rheological properties are analyzed next. The cases $\kappa=0$ and $\kappa \neq 0$ are treated separately.

\section{Stationary Solutions}

\subsection{Rheological Properties for $\kappa=0$}

\section{Implicit Solutions}

For $\kappa=0$ and for the isotropic potential which leads to (19) with (20), one has $\pi_{0}=0$ in the stationary state. Then (24) and (23) reduce to $\cos (2 \chi)=\pi_{c} p$ and $\pi_{c}^{-1} \Gamma p \sin (2 \chi)=\psi p^{2}$. These two equations provide the parametric representations $\chi(p)$ and $\Gamma(p)$ and thus allow to generate graphs showing the non-Newtonian viscosity and other rheological properties as functions of the shear rate. More specifically, one has for the viscosity and the shear rate

$$
\begin{aligned}
& H(p)=\left(1-\pi_{c}^{2} p^{2}\right)\left(A^{*}-4 p^{2}+3 p^{4}\right)^{-1} \\
& \Gamma(p)=\pi_{c} p\left(A^{*}-4 p^{2}+3 p^{4}\right)\left(1-\pi_{c}^{2} p^{2}\right)^{-1 / 2} .
\end{aligned}
$$

This implicit solution only exists for certain ranges for $p$, e.g. $0<p<\pi_{c}^{-1}$. In the following, curves are displayed for representative values of the parameter $A^{*}$.

These results should be compared with those based on the previously used anisotropic potential function which leads to (17) with (18). In this case one has $\pi_{0} \neq$ 0 even for $\kappa=0$. Here the implicit solution of the problem proceeds as follows $[1,8]$. The stationary solution of the relaxation equation for $\pi_{0}$, cf. (16), with $q=$ $\pi_{0}$, implies $p(q)^{2}=-q\left(A^{*}-3 q+q^{2}\right)(3+2 q)^{-1}$. Then (24) and (23) lead to the parametric representations $\chi(q)$ and $\Gamma(q)$ which are used to plot rheological properties as functions of the shear rate. In particular, one has for the viscosity and the shear rate $H(q)=$ $\left(1-\pi_{c}^{2} p(q)^{2}\right)\left(A^{*}+6 q+2\left(p(q)^{2}+q^{2}\right)^{-1}\right)$ and $\Gamma(q)=$ $\pi_{c} p(q)\left(A^{*}+6 q+2\left(p(q)^{2}+q^{2}\right)\right)\left(1-\pi_{c}^{2} p(q)^{2}\right)^{-1 / 2}$. Of course, $q$ has to be chosen in appropriate ranges, e. g. $-1.5<q<0$.

\section{Non-Newtonian Viscosity}

In Fig. 1 the non-Newtonian viscosity function $H=$ $H(s)+H_{\infty}$, where $s$ stands for either $p$ or $q$, is plotted as a function of the shear rate $\Gamma$ for $\pi_{c}=1, H_{\infty}=0.1$, and $A^{*}=2.0,2.5,4.0$. The first Newtonian viscosity, reached for $\Gamma \ll 1$, is $H_{\mathrm{New}}=\left(A^{*}\right)^{-1}+H_{\infty}$. It is equal to $0.6,0.5,0.35$ for the cases shown. The thicker 


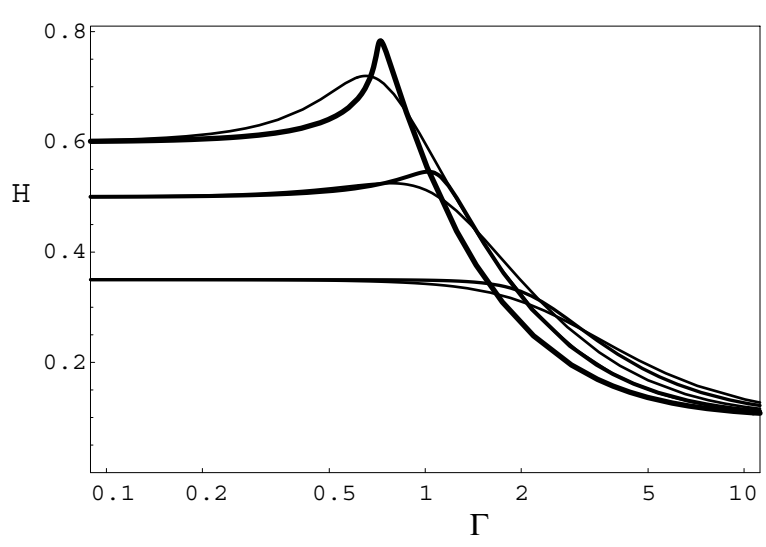

Fig. 1. The non-Newtonian viscosity $H$ versus the shear rate $\Gamma$ for $A^{*}=2.0,2.5,4.0$ (from top to bottom) and $\pi_{c}=$ 1.0, $\kappa=0.0, H_{\infty}=0.1$. A logarithmic scale is used for the horizontal axis. The thick and thin curves pertain to the isotropic and to the anisotropic potential functions, respectively.

curves pertain to the new isotropic potential, the thin curves are for the anisotropic potential used in $[1,8]$. A transition from a simple shear-thinning behaviour to a more complex shear-thickening behaviour followed by shear-thinning at higher shear rates occurs when the control parameter $A^{*}$ decreases.

For small shear rates the expansion of the nonNewtonian viscosity function $H$ with respect to $\Gamma$ yields $H=H_{\mathrm{New}}-h_{2}\left(A^{*}, \kappa\right) \Gamma^{2}+\ldots$. In the case of the isotropic potential one finds $h_{2}=\left(A^{*}\right)^{-3}\left(1-4\left(A^{*}\right)^{-1}\right)$ for $\pi_{c}=1$. Thus $h_{2}$ is negative and shear-thickening occurs for $A^{*}<4$. In the case of the anisotropic potential used in $[1,8]$, the corresponding expression is $h_{2}=\left(A^{*}\right)^{-3}\left(1+2\left(A^{*}\right)^{-1}-18\left(A^{*}\right)^{-2}\right)$. In this case shear-thickening occurs for $A^{*}<\sqrt{19}-1 \approx 3.36$. A discussion of the dependencies on $\kappa$ and $\pi_{c}$ is presented below.

\section{Shear Stress}

The shear stress $\sigma$ as given by (12) is displayed in Fig. 2 for the same values of the parameters as used above for the viscosity. Figure $2 \mathrm{~A}$ represents the isotropic and Fig. 2B the anisotropic potential functions, respectively. In the second case, the curve for $A^{*}=1.75$ is shown in addition.

For a certain range of the shear stress, not only one but more values of the shear rate pertain to one value of the shear stress. Here shear banding is expected to occur in the system, cf. [38]. The spontaneous formation of spatial inhomogeneities is not pursued here.
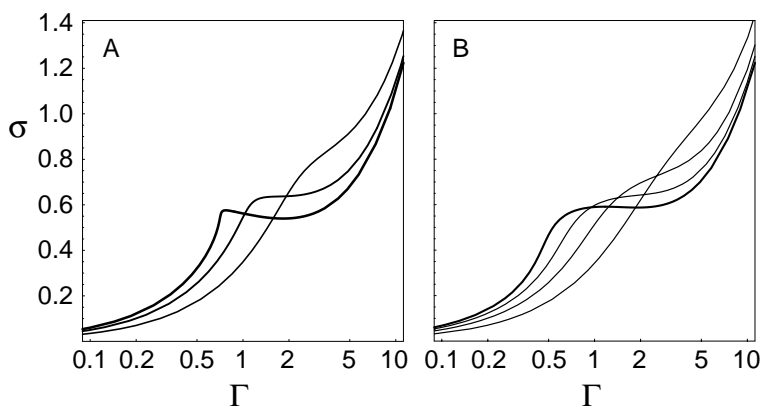

Fig. 2. The shear stress $\sigma$ versus the shear rate $\Gamma$ for $A^{*}=$ $2.0,2.5,4.0$ (from left to right) and $\pi_{c}=1.0, \kappa=0.0$, $H_{\infty}=0.1$. A logarithmic scale is used for the horizontal axis. (A) Isotropic potential function. (B) Anisotropic potential function; the thick curve (most left at small shear rates) is for $A^{*}=1.75$.

\section{Normal Stress Differences, Viscometric Functions}

The stress differences and viscometric functions can be computed in analogy to the shear stress and viscos-
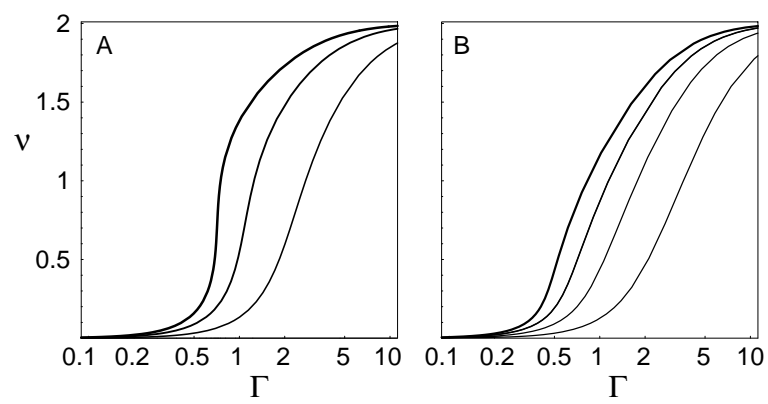

Fig. 3. The first normal stress difference $v=N_{1}$ versus the shear rate $\Gamma$ for $A^{*}=2.0,2.5,4.0$ (from left to right) and $\pi_{c}=1.0, \kappa=0.0$. A logarithmic scale is used for the horizontal axis. (A) Isotropic potential function. (B) Anisotropic potential function; the thick curve (most left at small shear rates) is for $A^{*}=1.75$.
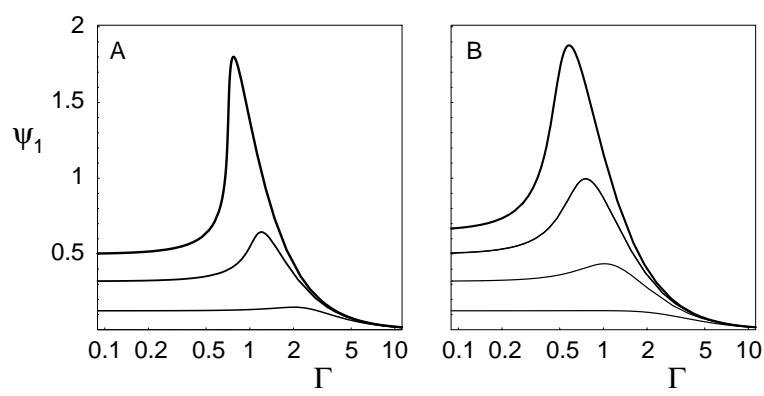

Fig. 4. The first viscometric function $\Psi_{1}$ versus the shear rate $\Gamma$. (A) Isotropic potential function. (B) Anisotropic potential function. The model parameters are the same as chosen for Figure 3. 

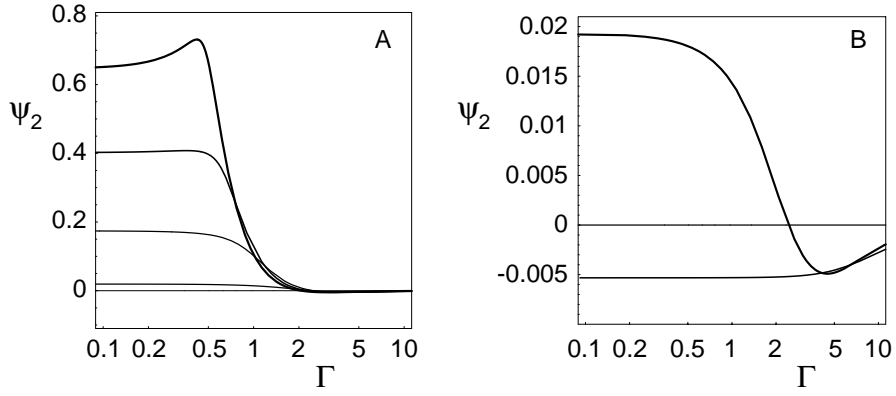

Fig. 5. The second viscometric function $\Psi_{2}$ versus the shear rate $\Gamma$ as obtained with the anisotropic potential functions. (A) $A^{*}=1.75,2.0,2.5,4.0$. (B) $A^{*}=4.0,8.0$. Notice the different vertical scales. ity. In Fig. 3 the first normal stress difference $v=N_{1}$ is displayed as a function of the shear rate (logarithmic scale) for the same values of the parameters as in Figure 2. Again Fig. 3A represents the isotropic and Fig. 3B the anisotropic potential functions, respectively. The parameters are $A^{*}=2.0,2.5,4.0$, and $\pi_{c}=$ $1.0, \kappa=0.0$. The thick curve in Fig. 3B (most left at small shear rates) is for $A^{*}=1.75$. The corresponding curves for the first viscometric function $\Psi_{1}$ are shown in Figure 4. The small shear rate limit of $\Psi_{1}$ is $2\left(A^{*}\right)^{-2}$. A shear-thickening-like behaviour is also seen for $\Psi_{1}$. In the case of the isotropic and anisotropic potentials this occurs for $A^{*}<8$ and $A^{*}<2(\sqrt{10}-1) \approx 4.325$, for $\pi_{c}=1$. These values are larger than those for the onset of shear-thickening revealed by the viscosity.

The behaviour of the shear stress, the first normal stress difference, as well as of the viscosity and the first viscometric function are qualitatively rather similar for the results pertaining to the isotropic and to the anisotropic potential functions. There are quantitative differences, however. The second normal stress difference and the second viscometric function, on the other hand, differ also qualitatively for both potentials. For the case of the isotropic potential one has $N_{2}=-(1 / 2) N_{1}<0$ and $\Psi_{2}=-(1 / 2) \Psi_{1}<0$. For the anisotropic potential, the small shear rate limit of $N_{2}$ and $\Psi_{2}$, with $\pi_{c}=1$, is negative or positive depending on whether $A^{*}$ is larger or smaller than $3 \sqrt{3} \approx 5.196$. At higher shear rates, $\Psi_{2}$ may change sign with increasing shear rate.

In Fig. 5 the second viscometric function $\Psi_{2}$ is plotted versus the shear rate $\Gamma$, as calculated with the anisotropic potential function. Figure $5 \mathrm{~A}$ is for $A^{*}=$ 1.75, 2.0, 2.5, 4.0, Fig. 5B for $A^{*}=4.0,8.0$. As before, $\pi_{c}=1.0$ is used.

\subsection{Rheological Properties for $\kappa \neq 0$}

The case of the isotropic potential is considered first. For a stationary situation, (23) and the relaxation equation for $\pi_{0}=q$ lead to $q\left(1-\tilde{\kappa} \pi_{c} q\right)+\tilde{\kappa} \pi_{c} p^{2}=0$. This relation is independent of the specific form of $\psi$, cf. (20). Now one can either express $p$ as a function of $q$, viz. $p=\sqrt{-\left(\tilde{\kappa} \pi_{c}\right)^{-1} q\left(1-\tilde{\kappa} \pi_{c} q\right)}$, or $q$ as a function of $p$, viz.

$$
q=\frac{1}{2}\left(\tilde{\kappa} \pi_{c}\right)^{-1}\left(1-\sqrt{1+4 p^{2} \tilde{\kappa}^{2} \pi_{c}^{2}}\right) .
$$

The sign is chosen such that $q=\pi_{0}$ is negative. This corresponds to the case where the principal direction associated with the largest eigenvalue of the stress tensor remains perpendicular to the vorticity direction, i. e. in the $x y$-plane, just as for a simple shear flow with $\kappa=0$. The shear rate and the other quantities of interest can be computed as functions of either $q$ or $p$. The use of $p$ as parameter is computationally more convenient for small values of $\kappa=\sqrt{3} \tilde{\kappa}$. In any case, $q \rightarrow-\tilde{\kappa} \pi_{c} p^{2}$ for small $q$ and $\tilde{\kappa} \rightarrow 0$. Thus $q=0$ is the stationary solution for $\tilde{\kappa}=0$, as presupposed above. Insertion of $q=q(p)$ given by (26) or of $p=p(q)$ into (24) yields $\chi$ as a function of $p$ or of $q$. From (23) one infers

$$
\Gamma=\pi_{c} p \psi\left(\pi^{2}\right)\left(1-\tilde{\kappa} \pi_{c} q\right)^{-1}(\sin (2 \chi))^{-1}
$$

with

$$
\psi\left(\pi^{2}\right)=A^{*}-4 \pi^{2}+3 \pi^{4}, \quad \pi^{2}=p^{2}+q^{2} .
$$

The shear stress, the viscosity as well as the normal pressure differences can be plotted as functions of the shear rate. Examples for the dependence of the nonNewtonian viscosity on $\kappa$ are displayed in Figure 6.

The implicit analytical stationary solution for the anisotropic potential employed in $[1,8]$ is obtained by computing the quantities of interest as functions of $q=\pi_{0}$. In the stationary state (23) now implies

$$
\psi_{1} p^{2}=\pi_{c}^{-1} \Gamma p \sin (2 \chi)\left(1-\tilde{\kappa} \pi_{c} q\right)
$$



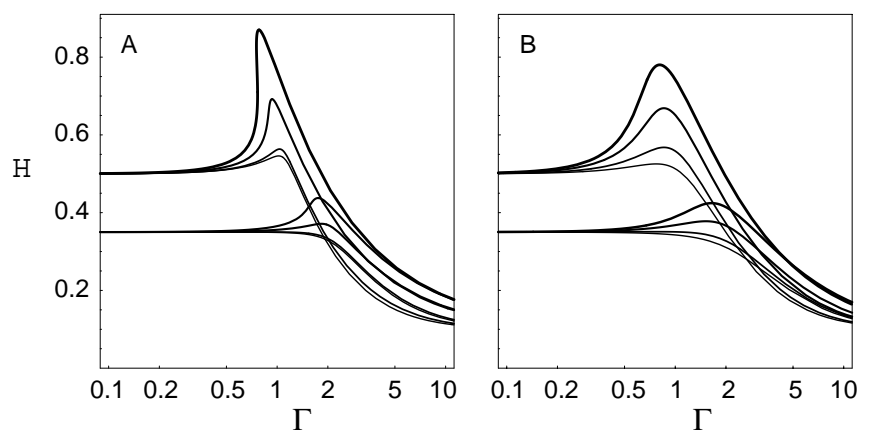

Fig. 6. The dependence of the non-Newtonian viscosity $H$ on the model parameter $\kappa$. The curves are for $\kappa=0.01,0.4,1.0$, and 1.5 , which yields the highest maxima. A logarithmic scale is used for the shear rate $\Gamma$. (A) Isotropic potential function. (B) Anisotropic potential function. The curves starting at 0.5 and 0.35 are for $A^{*}=2.5,4.0$, and $\pi_{c}=$ $1.0, H_{\infty}=0.1$, in all cases. with $\psi_{1}=A^{*}+6 q+2 q^{2}+2 p^{2}$. The stationary version of the relaxation equation (16) for $\pi_{0}=q$ is equivalent to

$$
\psi_{0} q+3 p^{2}+\tilde{\kappa} \Gamma p \sin (2 \chi)=0
$$

with $\psi_{0}=A^{*}-3 q+2 q^{2}+2 p^{2}$. For $\tilde{\kappa} \neq 0$ one has $\Gamma p \sin (2 \chi)=-\tilde{\kappa}^{-1}\left(\psi_{0} q+3 p^{2}\right)$. Insertion of this expression into (29) yields $\psi_{1} p^{2}+\pi_{c}^{-1} \tilde{\kappa}^{-1}\left(\psi_{0} q+\right.$ $\left.3 p^{2}\right)\left(1-\tilde{\kappa} \pi_{c} q\right)=0$. This is a quadratic equation for $p^{2}$ which can be solved for $p^{2}=p^{2}(q)$ :

$$
p^{2}(q)=\tilde{\kappa}^{-1} b(q)\left(\sqrt{1-\tilde{\kappa} c(q) / b(q)^{2}}-1\right),
$$

$b(q)=\left(\pi_{c}^{-1}\left(1-\tilde{\kappa} \pi_{c} q\right)(3+2 q)+\tilde{\kappa}\left(A^{*}+6 q+2 q^{2}\right)\right) / 4$,

$c(q)=\pi_{c}^{-1}\left(1-\tilde{\kappa} \pi_{c} q\right)\left(A^{*}-3 q+2 q^{2}\right) / 2$.

For $\tilde{\kappa} \rightarrow 0$ the expression (31) for $p^{2}$, with (32), reduces to the relation given above for $\kappa=0$. Now $p=p(q)$ can be inserted into (24) and (30) to obtain $\chi(q)$ and $\Gamma(q)$ in analogy to the cases considered before.

In Fig. 6 the dependence of the non-Newtonian viscosity $H$ on the model parameter $\kappa$ is presented. The curves are for $\kappa=0.01,0.4,1.0$, and 1.5 , which yields the highest maximum. As before, a logarithmic scale is used for the shear rate $\Gamma$. Figure 6A pertains to the isotropic and Fig. 6B to the anisotropic potential functions, respectively. The curves starting at 0.5 and 0.35 are for $A^{*}=2.5,4.0$, and $\pi_{c}=1.0, H_{\infty}=0.1$ were chosen in all cases. Clearly, the shear-thickening behaviour is stronger for larger values of $\kappa$.

\subsection{Shear-Thickening Phase Diagram}

Shear-thickening behaviour is indicated by a positive value of the derivative of the viscosity with respect to the shear rate in the small shear rate limit,
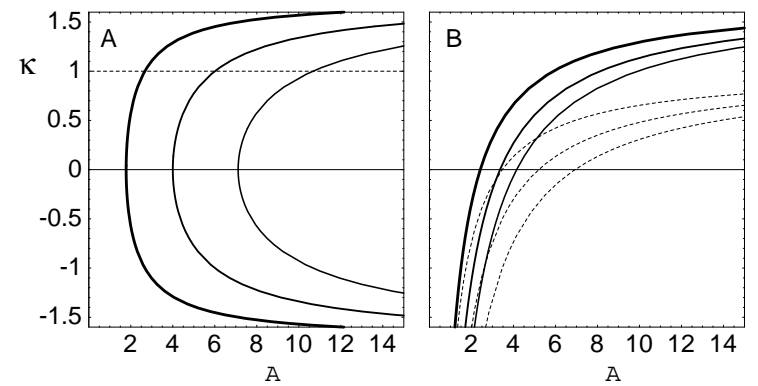

Fig. 7. Shear-thickening phase diagram in the $A^{*}-\kappa$-plane. Shear-thickening occurs for those values of $A^{*}$ and $\kappa$ which are on the left-hand side of the curves. These are drawn, from left to right, for $\pi_{c}=1.5,1.0,0.75$. (A) Isotropic potential function. (B) Anisotropic potential function. The dashed curves indicate the sign change of the second normal stress difference.

viz. by $\mathrm{d} H / \mathrm{d} \Gamma>0$ for $\Gamma \rightarrow 0$. For given parameters $\kappa$ and $\pi_{c}$ a characteristic value $A_{\text {th }}$ exists such that shear-thickening occurs for $A^{*}<A_{\mathrm{th}}$. In the case of the isotropic potential one has

$$
A_{\text {th }}=4\left(1-\kappa^{2} / 3\right)^{-1} \pi_{c}^{-2} .
$$

The corresponding expression for the anisotropic potential is

$$
\begin{aligned}
A_{\mathrm{th}}= & \left((3 / 2) \sqrt{3} \kappa \pi_{c}-1\right. \\
& \left.+\sqrt{1-3 \sqrt{3} \kappa \pi_{c}+18 \pi_{c}^{2}+(3 / 4) \kappa^{2}}\right) \\
& \cdot\left(1-\kappa^{2} / 3\right)^{-1} \pi_{c}^{-2}
\end{aligned}
$$

Relations (33) and (34) are used to generate shearthickening phase diagrams. Figure 7 indicates the parameter ranges in the $A^{*}-\kappa$-plane where shearthickening occurs. Figure $7 \mathrm{~A}$ represents the isotropic and Fig. 7B the anisotropic potentials, respectively. Shear-thickening occurs for those values of $A^{*}$ and $\kappa$ 
which are on the left-hand side of the curves. The curves shown pertain to $\pi_{c}=1.5,1.0,0.75$. The dashed curves in Fig. 7 mark the regions where, in the limit of small shear rates, the ratio of the second and first normal stress differences is positive (above) or negative (below the curves).

\subsection{Normal Stress Differences at Small Shear Rates}

Let $v_{21}$ be the ratio of the second to the first normal stress difference $N_{2} / N_{1}=\psi_{2} / \psi_{1}$ in the limit of small shear rate, viz. for $\Gamma \rightarrow 0$. One finds $v_{21}=-(1 / 2)(1-$ $\kappa)$ and $v_{21}=-(1 / 2)(1-\kappa-3 \sqrt{3}) /\left(A^{*} \pi_{c}\right)$ for the isotropic and the anisotropic potential functions, respectively. The dashed curves in Fig. 7 indicate where $v_{12}$ changes sign. In particular, one has $v_{12}>0$ $\left(v_{12}<0\right)$ above (below) the dashed curves.

For the application of the model to a specific substance, i. e. for a decision between the different potential functions and a determination of the relevant model parameters, it is desirable to have data not only for the shear rate dependence of the viscosity but also for the the first and second normal stress differences. When only viscosity data are available, different sets of model parameters are possible. One example is presented next.

\subsection{Comparison with Experimental Data}

To demonstrate that the flow curves obtained within the present theoretical approach indeed are similar

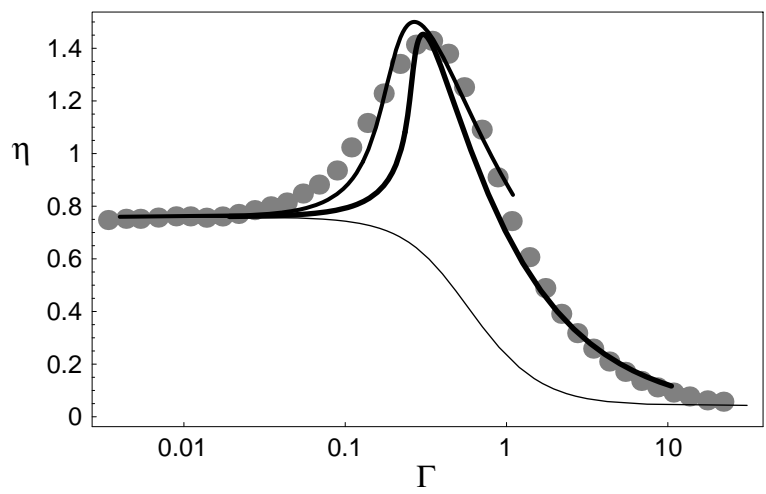

Fig. 8. Comparison of an experimental flow curve showing shear-thickening and shear-thinning behaviour with theoretical results. The data stem from [5]. The viscosity (linear scale) is in units of $\mathrm{Pa}$, the shear rate (logarithmic scale) in $\mathrm{s}^{-1}$. The model parameters and the reference values for the viscosity and the shear rate used for the theoretical curves are given in the text.

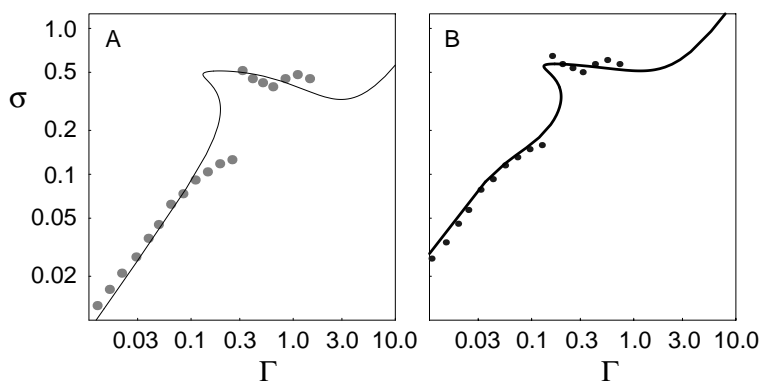

Fig. 9. Reduced shear stress versus reduced shear rate: comparison of experimental data (points) with theoretical results (curves). The data stem from [7].

to experimental findings, first a comparison is made with data presented in [5]. The measurements were performed on a system built up from an oil-in-water droplet microemulsion into which a telechelic polymer was incorporated. In Fig. 8 the experimental data, viscosity (in Pa) versus shear rate (in $\mathrm{s}^{-1}$, logarithmic scale), are represented by dots. The thick and thin curves pertain to the isotropic and anisotropic potential functions. For the thick curve, the reference viscosity $6 \mathrm{~Pa}$ was used, the dimensionless shear rate $\Gamma$ was multiplied by the reference shear rate $\dot{\gamma}_{\text {ref }}=5 \mathrm{~s}^{-1}$ in order to match approximately the shear rates of [5]. The model parameters for the thick curve are $A^{*}=8$, $\pi_{c}=1, H_{\infty}=0.01 / 6 \approx 0.002, \kappa=1.75 \sqrt{3} \approx 3.0$. For the thinner curves computed with the anisotropic potential function, the reference viscosity $4.2 \mathrm{~Pa}$ was chosen and the reference shear rate $\dot{\gamma}_{\text {ref }}=4.5 \mathrm{~s}^{-1}$ was used. Here the parameters are $A^{*}=6, \pi_{c}=1$, $H_{\infty}=0.01 / 4.2 \approx 0.002$, furthermore $\kappa=1.75 \sqrt{3} \approx$ 3.0 and $\kappa=0.002$ for the curves which show and do not show shear-thickening behaviour. It must be stressed that no search for the parameters was conducted which give the best fit.

Here the parameter $A^{*}$ is relatively large such that the shear-thickening behaviour is mainly due to $\kappa \neq 0$ as in the Johnson-Segalman model [32]. This should be different at lower temperatures or at higher densities where $A^{*}$ is smaller. More dramatic effects are expected at $A^{*} \approx 1$, i. e. close to solidification or gelation.

Next, it is indicated that also the discontinuous behaviour as found in surfactant solutions can be modeled by the present approach. The data stem from experiments conducted with controlled shear rate [7]. For simplicity, the comparison is restricted to the model with the anisotropic potential function and the fixed parameter values $\kappa=0, \pi_{c}=1.0$. In Fig. 9A the exper- 
imental values for the shear stress and the shear rate are divided by the reference values $\sigma_{\text {ref }}=79 \mathrm{~Pa}$ and $\dot{\gamma}_{\text {ref }}=251 \mathrm{~s}^{-1}$. The curve is computed from the stationary solution discussed above with $A^{*}=1.25, H_{\infty}=$ 0.05. The curve in Fig. 9B is for $A^{*}=1.25, H_{\infty}=0.15$. Here an additional contribution to the shear stress, viz. $H_{2} \Gamma /\left(1+\left(\tau_{2} \Gamma\right)^{2}\right)$, is included in order to account for the shear-thinning behaviour prior to the discontinuous shear-thickening. The values of the additional parameters are $H_{2}=2.0, \tau_{2}=15$. In this case the experimental data have been scaled with $\sigma_{\text {ref }}=63 \mathrm{~Pa}$ and $\dot{\gamma}_{\text {ref }}=501 \mathrm{~s}^{-1}$. It is not uncommon that rheological data are fitted by a superposition of Maxwell models with different relaxation times. The charm of the simplicity of model then is lost, however. Again, it must be stressed that it is not our intention to find a best fit for the data of a specific substance but to demonstrate the qualitative similarity between experimental findings and results inferred from the nonlinear Maxwell model.

\section{Yield Stress and Periodic Response}

\subsection{Yield Stress}

As mentioned above, the relaxation equation for the symmetric traceless stress tensor possesses non-zero solutions even in the absence of a flow when the parameter $A^{*}$ is below $4 / 3 \approx 1.333$ and $9 / 8=1.125$ for the isotropic and the anisotropic potential functions considered here. This yield stress or residual stress is typical for a solid-like material, crystalline or amorphous. The dependence of the yield stress on the model parameter $A^{*}$, which is recalled to decrease with decreasing temperature and with increasing density, is shown in Fig. 10 for $\pi_{c}=1$. The thin curve $((3+$ $\left.\left.\sqrt{9-8 A^{*}}\right) / 4\right)$ and the dashed curve $\left(-A^{*} / 2\right)$ pertain to uniaxial and planar biaxial stresses for the anisotropic potential. The thick curve $\left(\sqrt{\left(2+\sqrt{4-3 A^{*}}\right) / 3}\right)$ indicates the yield stress for the isotropic potential, irrespective of the stress symmetry.

For small deformations $\varepsilon=\Gamma t$ and small shear rates $\Gamma$, the model implies an elastic behaviour characterized by $\sigma_{x y}=G_{\text {eff }} \varepsilon$, where the effective shear modulus is given by $G_{\text {eff }}=G\left(1-\pi_{c} \tilde{\kappa} \pi_{0}^{\mathrm{ini}}\right)$. Here $G$ is the reference shear modulus introduced in (1) and $\pi_{0}^{\text {ini }}$ is the " 0 " component of the stress in the initial state. A detailed discussion of the elastic behaviour is outside of the scope of the present article. For larger deformations, typically $\varepsilon>1$, plastic flow results.

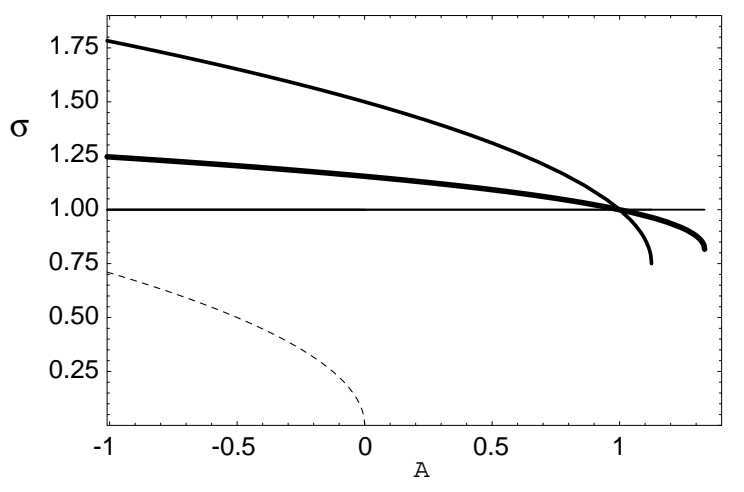

Fig. 10. The dependence of the yield stress on the model parameter $A^{*}$, for $\pi_{c}=1$. The thin curve and the dashed curve pertain to uniaxial and planar biaxial stresses for the anisotropic potential, the thick curve is for the isotropic potential.

Plastic flow and shear-induced melting occur in solid friction processes. These phenomena have been studied in Non-Equilibrium-Molecular-Dynamics (NEMD) computer simulations $[39,40]$. The present theory provides a generic model for the flow of solids. In many cases, analytical and numerical solutions of the nonlinear Maxwell model yield stationary solutions for a steady flow. However, there are parameter ranges where the stress shows a non-stationary response typical for stick-slip-like motions. The analogy with the behaviour of the alignment of nematic liquid crystals is useful.

\subsection{Stick-Slip Motions}

In nematic liquid crystals, the alignment tensor may be stationary or not in the presence of a steady shear flow depending on whether the "tumbling parameter" $\lambda$, defined for small shear rates, is larger or smaller than 1 [16]. For $\lambda<1$ the axis associated with the largest eigenvalue of the relevant tensor undergoes a periodic tumbling motion. At larger shear rates more complex and even chaotic motions [19,29-31,41], as well as a transition to a stationary state can occur. A similar behaviour, viz. a time-dependent shear stress as response to a stationary imposed velocity gradient, corresponding to a stick-slip-like motion, is found for the nonlinear Maxwell model. In [8] this was studied for the anisotropic potential. Comparison with the relaxation equation governing the alignment tensor shows that, for the anisotropic potential function, the parameter $\pi_{c}$ is related to the model parameter $\lambda_{K}$ of [29] by $\lambda_{K}=2 /\left(\sqrt{3} \pi_{c}\right)$. 

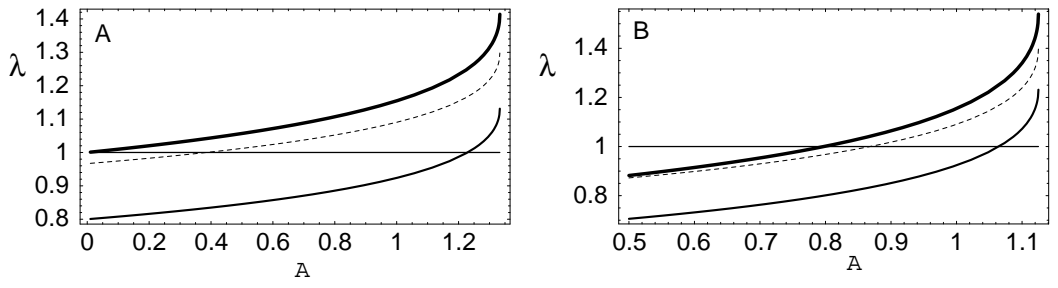

Fig. 11. The stick-slip parameter $\lambda$ versus $A^{*}$. (A) Isotropic potential function. (B) Anisotropic potential function. The parameters are $\left(\kappa, \pi_{c}\right)=(0.0,1.0),(0.0,1.25)$, and $(0.5,1.25)$ for the thick, the thin, and the dashed curves. The horizontal line marks the limit $\lambda=1$.
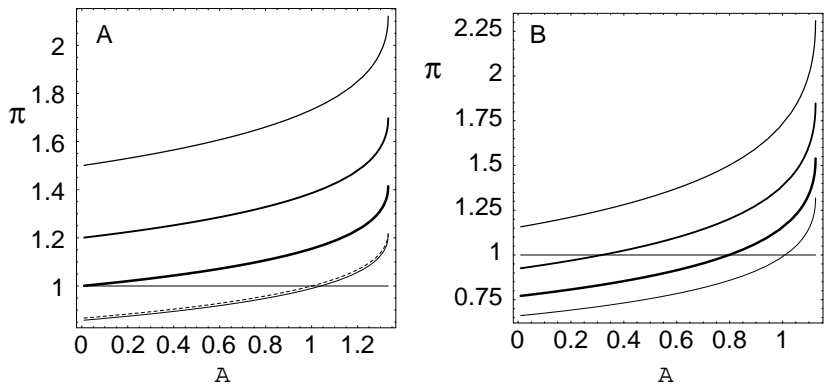

Fig. 12. Stick-slip phase diagram in the $A^{*}-\pi_{c}$-plane for $\kappa=-0.5,0.0,0.5,1.0$, from bottom to top. (A) Isotropic potential function. (B) Anisotropic potential function. Stick-slip like behaviour is expected for values of $\pi_{c}$ above the curves shown. The horizontal line marks $\pi_{c}=1$. The dashed curve in (A) corresponds to the planar biaxial case discussed in the text.
The "tumbling" or "stick-slip parameter" $\lambda$ is inferred from the expression (24) for the flow angle $\chi$, according to $\cos (2 \chi)=\lambda^{-1}$, in the limit of small shear rates and for those values of $A^{*}$ for which residual stresses and a yield stress exist. With $\pi_{0}=q$, one has

$$
\lambda=\left(\pi_{c} p^{\mathrm{eq}}\right)^{-1}-\tilde{\kappa}\left(q^{\mathrm{eq}} / p^{\mathrm{eq}}\right) .
$$

The superscript eq indicates the equilibrium values in the limit of vanishing shear rates. For a uniaxial stress tensor the components $p^{\mathrm{eq}}$ and $q^{\mathrm{eq}}$ are related to the principal value $\pi_{\mathrm{eq}}$ by

$$
p^{\mathrm{eq}}=(\sqrt{3} / 2) \pi_{\mathrm{eq}}, \quad q^{\mathrm{eq}}=-\pi_{\mathrm{eq}} / 2,
$$

and consequently

$$
\lambda=2\left(\sqrt{3} \pi_{c} \pi_{\mathrm{eq}}\right)^{-1}+\kappa / 3 .
$$

For the isotropic and the anisotropic potential functions one has $\pi_{\mathrm{eq}}=\sqrt{\left(2+\sqrt{4-3 A^{*}}\right) / 3}$ and $\pi_{\mathrm{eq}}=$ $\left(3+\sqrt{9-8 A^{*}}\right) / 4$, respectively. Hence the stick-slip parameter is only defined for $A^{*}<4 / 3 \approx 1.33$ and $A^{*}<9 / 8=1.125$ in these cases. The isotropic potential allows also a planar biaxial equilibrium solution. Then one has $p^{\mathrm{eq}}=\pi_{\mathrm{eq}}, q^{\mathrm{eq}}=0$, and (35) leads to $\lambda=\left(\pi_{c} \pi_{\mathrm{eq}}\right)^{-1}$, which is independent of the parameter $\kappa$.

In Fig. 11 the stick-slip parameter $\lambda$, as given by (37), is plotted versus $A^{*}$. Figures $11 \mathrm{~A}$ and B represents the isotropic and anisotropic potential functions, respectively. Notice the different horizontal scales. The model parameters are $\left(\kappa, \pi_{c}\right)=(0.0,1.0),(0.0,1.25)$, and $(0.5,1.25)$ for the thick, the thin, and the dashed curves, respectively. The horizontal line marks the limit 1 below which stick-slip-like behaviour is expected.

When $\lambda$ is put equal to 1 in (37), one obtains a stick-slip phase boundary in the $A^{*}-\kappa-\pi_{c}$-parameterspace separating stationary solutions from stick-sliplike ones. The intersections for different values of $\kappa$ yield a stick-slip phase diagram in the $A^{*}-\pi_{c}$-plane as displayed in Fig. 12, for $\kappa=-0.5,0.0,0.5,1.0$. Figures $12 \mathrm{~A}$ and $\mathrm{B}$ pertain to the isotropic and anisotropic potential functions, respectively. Stick-slip-like behaviour is expected for values of $\pi_{c}$ above the curves shown. The horizontal line $\pi_{c}=1$ marks the value which has been used in many calculations, here and in [8]. The dashed curve in Fig. 12A indicates $\pi_{c}=$ $\left(p^{\mathrm{eq}}\right)^{-1}$ corresponding to the planar biaxial case discussed above.

\subsection{Examples for Non-Stationary Response}

Examples for a time-dependent response of the shear stress and for the normal stress differences are displayed in Fig. 13 and Fig. 14 for the isotropic and anisotropic potential functions. The values for the parameter $\kappa$ are -0.5 and 0.5 , respectively. The other model parameters are $\left(A^{*}, \pi_{c}, H_{\infty}\right)=(0.5,2 / \sqrt{3} \approx$ $1.1547,1.0)$, and the imposed shear rate is $\Gamma=0.75$, in both cases. The inital values of all five com- 

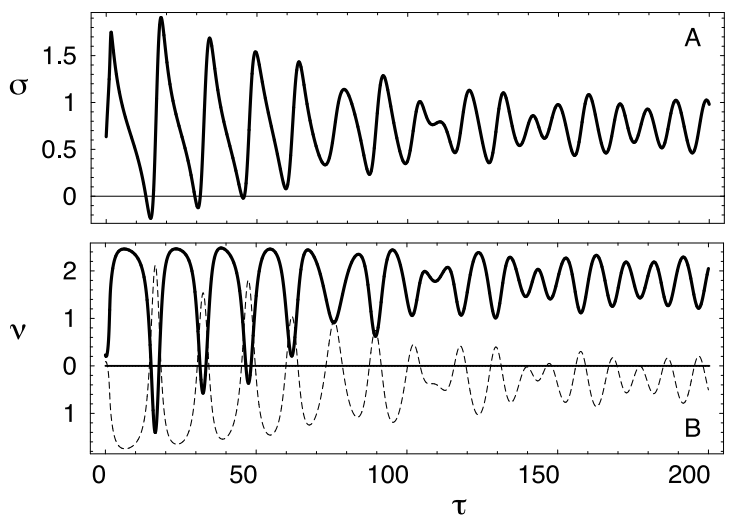

Fig. 13. The shear stress and the first and second (dashed curve) normal stress differences as functions of the time for the isotropic potential. The parameters are $\left(A^{*}, \pi_{c}, \kappa, H_{\infty}\right)=$ $(0.5,2 / \sqrt{3} \approx 1.1547,-0.5,1.0)$. The imposed shear rate is $\Gamma=0.75$.
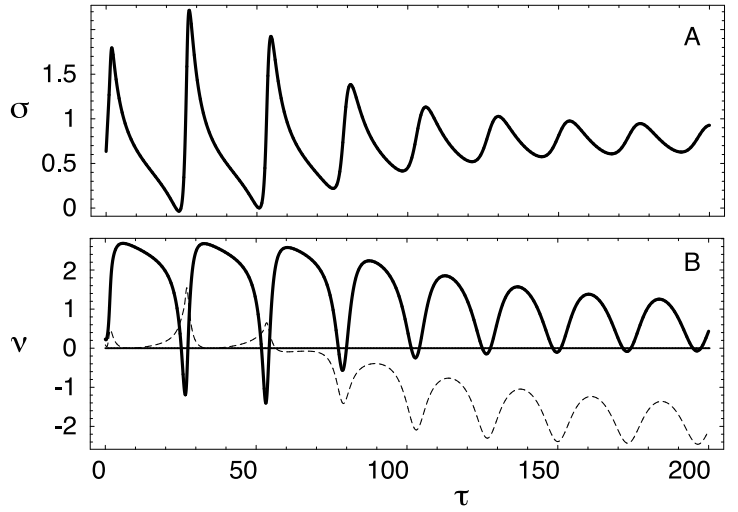

Fig. 14. The shear stress and the normal stress differences as functions of the time for the anisotropic potential. The parameters are $\left(A^{*}, \pi_{c}, \kappa, H_{\infty}\right)=(0.5,2 / \sqrt{3} \approx$ $1.1547,0.5,1.0)$. The imposed shear rate is $\Gamma=0.75$.

ponents of the tensor are small $( \pm 0.1)$, but nonzero.

A large-amplitude stick-slip-like behaviour is seen for about 100 time units with the amplitude of symmetry-breaking $x z$ - and $y z$-components grows to its final size, then the shear stress shows a smoother periodic behaviour. In liquid crystal terminology, the dynamics is initially of tumbling type where the principal axis associated with the largest eigenvalue of the tensor undergoes a periodic motion in the $x y$-plane. Later it turns into a kayaking-tumbling motion [42] where this principal axis performs a rotation about the $z$ (vorticity)-axis.

The stick-slip parameter as given by (37) for a uniaxial yield stress is $\lambda \approx 0.75$ and $\lambda \approx 0.93$ for the specific parameters chosen for the isotropic and the anisotropic potentials, respectively. Thus a periodic behaviour, at small shear rates, is definitely expected in both cases, and it is indeed observed. The shear stress shown in A of Fig. 13 and Fig. 14 are qualitatively rather similar for both cases. The differences are larger for the first and second (dashed curves) normal stress differences, displayed in the lower graphs.

The time dependence of the shear stress is qualitatively similar to that one found in NEMD computer simulations for the plastic flow of solids and in sliding friction $[39,40]$. The friction force observed in experiments shows a similar behaviour [43].

It should be mentioned again that the discussion of the full complexity of the rich dynamic behaviour of the nonlinear Maxwell model is outside the scope of the present article. Further examples for timedependent responses are shown in $[8,44]$.

\section{Concluding Remarks}

The stability of solutions as discussed here was studied for the isotropic potential function [45], a bifurcation analysis for the anisotropic potential case has been performed recently [44]. These aspects are outside the scope of article. The present approach is based on a symmetric traceless extra stress tensor. As a generalization, scalars, like the trace of the pressure tensor or the density of the fluid, can be treated as additional dynamic variables. Related models which led to a chaotic behaviour were studied in $[38,46]$. The dynamics of two coupled second-rank tensors were used to treat side-chain liquid-crystalline polymers [47].

An extension of the theory to magneto-rheological fluids is possible if additional terms depending on an external magnetic field $\mathbf{B}$ are taken into consideration in the potential function. The simplest terms of this kind are $b_{1} \mathbf{B} \cdot \boldsymbol{\pi} \cdot \mathbf{B}+(1 / 2) b_{2} \mathbf{B} \cdot{ }^{\prime} \overline{\boldsymbol{\pi} \cdot \boldsymbol{\pi}} ' \cdot \mathbf{B}$ with the phenomenological coupling coefficients $b_{1}$ and $b_{2}$. Then the tensor $\boldsymbol{\Phi}(\boldsymbol{\pi})$ in the relaxation equation (2) for the stress tensor is replaced by

$$
\boldsymbol{\Phi}(\boldsymbol{\pi})+b_{1}{ }^{\mathrm{BB}}+b_{2} \overline{\overline{\mathbf{B B}}^{\prime}} \cdot \boldsymbol{\pi} .
$$

Due to $b_{2} \neq 0$, the rheological properties, in particular the viscosity and the viscometric functions, depend on the magnitude and the direction of the applied field. Most frequently the field is chosen in the direction of the velocity gradient [48]. The term involving $b_{1}$ leads 
to a normal stress difference, even in the absence of a flow. This solid-like behaviour has been observed in high-density colloidal dispersions of magnetic particles [49]. A more detailed analysis is desirable, preliminary results are available [45].

Finally, it must be stressed that the analysis of the model equation was restricted to the plane Couette geometry with an imposed shear rate. The gradient of the flow velocity has essentially been treated like an external field. In general, one has to deal with spatial inhomogeneities and the normal stress differences will set up secondary flows. The equations for the components of the stress tensor, cf. (16), then contain all three components of the vorticity and all five components of the strain rate tensor rather than just the $z$ - and the $x y$-components, viz. $\Omega$ and $\Gamma$, respectively. The full hydrodynamic problem has to be solved, viz. the local conservation equation for the linear momentum $\rho \frac{\mathrm{d} v_{\mu}}{\mathrm{d} t}+\nabla_{\mu} P=\nabla_{\nu} \sigma_{v \mu}$ and the dynamic equation (2) for the stress tensor $\sigma_{\mu v}$. Here $P$ is the (hydrostatic) pressure. The application of methods used for the study of the spatio-temporal behaviour of lasers [50] and for

[1] O. Hess and S. Hess, Physica A 207, 517 (1994); S. Hess and O. Hess, Nonlinear Flow Behaviour of Fluids, in: Nonlinear Thermodynamical Processes in Continua, TUB-Dokumentation 61a (Eds. W. Muschik and G. A. Maugin), TUB, Berlin 1992, p. $122-134$; O. Hess and S. Hess, Generalized Maxwell Model Equations for the Static and Dynamic Nonlinear Flow Behaviour, in: STATPHYS, 19th ed. (Ed. H. Bailin), World Scientific, Singapore 1996, pp. 369-382.

[2] H. A. Barnes, J. Rheol. 33, 329 (1989).

[3] H. M. Laun, R. Bung, S. Hess, W. Loose, O. Hess, K. Hahn, E. Hädicke, R. Hingmann, F. Schmidt, and P. Lindner, J. Rheol. 36, 743 (1992).

[4] J. Mewis and G. Biebaut, J. Rheol. 45, 799 (2001).

[5] E. Michel, J. Appell, F. Molino, J. Kiefer, and G. Porte, J. Rheol. 45, 1465 (2001).

[6] P. Fischer, E. K. Wheeler, and G. G. Fuller, Rheol. Acta 41, 25 (2002).

[7] R. Landolt and P. Fischer, Proceedings of the 6th European Conference on Rheology (Eds. H. Münstedt, J. Kaschta, and A. Merten), Erlangen 2002, pp. 559560.

[8] O. Hess, C. Goddard, and S. Hess, Physica A 366, 31 (2006).

[9] S. Hess, Z. Naturforsch. 30a, 728, 1224 (1975).

[10] S. Hess, Z. Naturforsch. 31a, 1034 (1976).

[11] M. Doi, J. Polym. Sci. Polym. Phys. Ed. 19, 229 (1981). the flow of liquid crystals [51] seems to be promising. Results are available for a three-dimensional flow problem [52]. Alternatively, it is feasible to generalize the smooth particle dynamics calculations of [53] where the symmetric traceless friction stress tensor was treated as an independent variable obeying a corotational Maxwell model to the case of the nonlinear model studied here.

\section{Acknowledgements}

Partial financial support by the Deutsche Forschungsgemeinschaft (DFG) via the project He1108/8 - 1 "Rheologie und stationäre Nichtgleichgewicht-Strukturen von Modell-Ferrofluiden" of the Forschungsschwerpunkt SPP 1104 "Kolloidale magnetische Flüssigkeiten: Grundlagen, Entwicklung und Anwendung neuartiger Ferrofluide" is gratefully acknowledged. Further support was given by the DFG via the Sonderforschungsbereich 448 "Mesoskopisch strukturierte Verbundsysteme". Support via an EPSRC Doctoral Training Grant is gratefully acknowledged.

[12] S. Hess and I. Pardowitz, Z. Naturforsch. 36a, 554 (1981).

[13] P. D. Olmsted and P. Goldbart, Phys. Rev. A 41, 4578 (1990); Phys. Rev. A 46, 4966 (1992).

[14] C. Pereira Borgmeyer and S. Hess, J. Non-Equilib. Thermodyn. 20, 359 (1995).

[15] B. J. Edwards, J. Non-Equilib. Thermodyn. 27, 5 (2002).

[16] R. G. Larson, The Structure and Rheology of Complex Fluids, Oxford University Press, Oxford, UK 1999.

[17] M. Kröger, Phys. Rep. 390, 453 (2004).

[18] W. Muschik and B. Su, J. Chem. Phys. 107, 580 (1997).

[19] M. G. Forest, Q. Wang, and R. Zhou, Rheol. Acta 44, 80 (2004).

[20] G. A. Maugin and W. Muschik, J. Non-Equilib. Thermodyn. 19, 217, 250 (1994).

[21] L. Restuccia and G. A. Kluitenberg, Physia A 154, 157 (1988); J. Non-Equilib. Thermodyn. 15, 335 (1990).

[22] M. Francaviglia, L. Restuccia, and P. Rogolino, J. NonEquilib. Thermodyn. 29, 221 (2004).

[23] S. Grandner, S. Heidenreich, P. Ilg, S. Klapp, and S. Hess, Phys. Rev. E 75, 040701 (2007).

[24] N. Herdegen and S. Hess, Physica A 115, 281 (1982).

[25] W. Loose and S. Hess, Phys. Rev. Lett. 58, 2443 (1988); Phys. Rev. A 37, 2099 (1988).

[26] S. Hess, Physica A 118, 79 (1983); in: Rheological Modelling: Thermodynamical and Statistical Approaches (Eds. J. Casas-Vázques and D. Jou), Lecture 
Notes in Physics 381, Springer, Berlin 1991, p. 51; J. C. Rainwater and S. Hess, Physica A 118, 371 (1983).

[27] H.C. Öttinger, Beyond Equilibrium Thermodynamics, Wiley Interscience, Hoboken, New Jersey, USA 2005.

[28] S. Heidenreich, P. Ilg, and S. Hess, Phys. Rev. E 73, 061710 (2006)

[29] G. Rienäcker, M. Kröger, and S. Hess, Phys. Rev. E 66, 040702(R) (2002); Physica A 315, 537 (2002).

[30] S. Hess and M. Kröger, Regular and Chaotic Rheological Behaviour of Tumbling Polymeric Liquid Crystals, in: Computer Simulations Bridging Liquid Crystals and Polymers (Eds. P. Pasini, C. Zannoni, and S. Zumer), Kluwer, Dordrecht 2004.

[31] S. Hess and M. Kröger, J. Phys.: Condens. Matter 16, S3835 (2004).

[32] M. W. Johnson and D. Segalman, J. Non-Newt. Fluid Mech. 2, 225 (1977)

[33] O. Rodulescu and P. Olmsted, Non-Newt. Fluid Mech. 91, 143 (2000).

[34] H.J. Wilson and S. M. Fielding, Non-Newt. Fluid Mech. 138, 181 (2006).

[35] S. Hess, Phys. Rev. A 25, 6141 (1982).

[36] H. Giesekus, Rheol. Acta 5, 29 (1966); J. Non-Newt. Fluid Mech. 11, 69 (1982).

[37] P. Kaiser, W. Wiese, and S. Hess, J. Non-Equilib. Thermodyn. 17, 153 (1992).

[38] S. M. Fielding and P. D. Olmsted, Phys. Rev. Lett. 90, 224501 (2003); 92, 084502 (2004); Eur. Phys. J. E 11, 65 (2003).

[39] S. Hess and M. Kröger, Phys. Rev. E 64, 011201 (2001); Techn. Mech. 22, 79 (2002).

[40] M. Kröger, I. Stankovic, and S. Hess, Multiscale Model. Simul. 1, 25 (2003); I. Stankovic, S. Hess, and M. Kröger, Phys. Rev. E 69, 021509 (2004); Phys. Rev. E 70, 066139 (2004).
[41] M. Grosso, R. Keunings, S. Crescitelli, and P. L. Maffettone, Phys. Rev. Lett. 86, 3184 (2001).

[42] R. G. Larson and H. C. Öttinger, Macromolecules 24, 6270 (1991).

[43] H. Yoshizawa, Y.-L. Chen, and J. Israelachvili, Wear 168, 161 (1993).

[44] C. Goddard, O. Hess, A. Balanov, and S. Hess, Phys. Rev. E 77, 026311 (2008).

[45] B. Arlt, Consequences of a Generalized Maxwell Model for Nonlinear Flow, Diplomarbeit, TU Berlin 2007.

[46] M.E. Cates, D. A. Head, and A. Ajdari, Phys. Rev. E 66, 025202 (2002).

[47] S. Hess and P. Ilg, Rheol. Acta 44, 465 (2005); P. Ilg and S. Hess, J. Non-Newt. Fluid Mech. 134, 2 (2006).

[48] S. Odenbach, J. Phys.: Condens Matter 15, S1497 (2003); C. Rinaldi, A. Chaves, S. Elborai, X. He, and M. Zahn, Curr. Opin. Colloid Interface Sci. 10, 141 (2005).

[49] H. See and R. Tanner, Rheol. Acta 42, 166 (2003); H. M. Laun, C. Gabriel, and G. Schmidt, J. Non-Newtonian Fluid Mech. 148, 47 (2008).

[50] E. Gehrig and O. Hess, Spatio-Temporal Dynamics and Quantum Fluctuations of Semiconductor Lasers, Springer, Heidelberg 2003.

[51] R. Zhou, M. G. Forest, and Q. Wang, Multiscale Model Simul. 3, 853 (2005); M. G. Forest, S. Heidenreich, S. Hess, X. Yang, and R. Zhou, J. Non-Newtonian Fluid Mech. 155, 130 (2008).

[52] C. Goddard, Rheological Chaos and Elastic Turbulence in a Generalized Maxwell Model for Viscoelastic Fluid Flow, Thesis, University of Surrey, Guildford, England 2008.

[53] M. Ellero, M. Kröger, and S. Hess, J. Non-Newt. Fluid Mech. 105, 35 (2002). 